\title{
Self-assembly of quantum dots and rings by droplet epitaxy and their optical properties
}

\author{
Takaaki Mano, ${ }^{a}$ Takashi Kuroda, ${ }^{b}$ Keiji Kuroda, ${ }^{c}$ and Kazuaki Sakodad \\ ${ }^{\text {a }}$ Quantum Dot Research Center, National Institute for Materials Science, 1-2-1 Sengen, \\ Tsukuba, Ibaraki 305-0047, Japan \\ mano.takaaki@nims.go.jp \\ ${ }^{\mathrm{b}}$ Quantum Dot Research Center, National Institute for Materials Science, 1-1 Namiki, \\ Tsukuba, Ibaraki 305-0044, Japan \\ PREST, Japan Science and Technology Agency (JST), 4-1-8 Honcho, Kawaguchi, Saitama \\ 332-0012, Japan \\ kuroda.takashi@nims.go.jp \\ ${ }^{c}$ Quantum Dot Research Center, National Institute for Materials Science, 1-1 Namiki, \\ Tsukuba, Ibaraki 305-0044, Japan \\ Present address: Department of Physics, School of Science, Kitasato University, 1-15-1 \\ Kitazato, Sagamihara, Kanagawa 228-8555, Japan \\ kkuroda@kitasato-u.ac.jp \\ ${ }^{\mathrm{d}}$ Quantum Dot Research Center, National Institute for Materials Science, 1-1 Namiki, \\ Tsukuba, Ibaraki 305-0044, Japan \\ Graduate School of Pure and Applied Sciences, University of Tsukuba, 1-1-1 Tennodai, \\ Tsukuba, Ibaraki 305-8577, Japan \\ sakoda.kazuaki@nims.go.jp
}

\begin{abstract}
Developments in the self-assembly by droplet epitaxy in our research group have enabled us to fabricate various GaAs quantum nanostructures of high optical quality such as quantum dots, single quantum rings, and concentric double quantum rings. We clarified their electronic states and relaxation processes by micro photoluminescence experiments. We achieved lasing of lattice-matched GaAs quantum dots and their excitonic Rabi oscillation by resonant excitation. We succeeded in the control of their photon emission rate by photonic crystal micro cavities.
\end{abstract}

Keywords: droplet epitaxy, quantum dot, quantum ring, GaAs, Purcell effect, lasing.

\section{INTRODUCTION}

In this article, we review recent developments in the self-assembly of quantum confined nanostructures by droplet epitaxy, or the Koguchi method [1-3]. Although the StranskiKrastanow (S-K) growth mode is often used for such self-assembly [4, 5], it can only be applied to lattice-mismatched systems like $\operatorname{In}(\mathrm{Ga}) \mathrm{As} / \mathrm{GaAs}$ for which the lattice constant of the three-dimensional nanostructure $(\operatorname{In}(\mathrm{Ga}) \mathrm{As})$ is different from that of the substrate $(\mathrm{GaAs})$. On the other hand, the droplet epitaxy is applicable not only to the lattice-mismatched systems $[6,7]$ but also to lattice-matched systems like GaAs/AlGaAs [8]. The lattice-matched systems are strain free in principle, and hence, have simpler and more isotropic electronic structures than the lattice-mismatched systems. This property often simplifies the interpretation of fundamental optical experiments.

As we shall show in this article, we can create various structures such as quantum dots (QDs) [6-10], single quantum rings (single-Qrings) [11], and concentric double quantum rings (double-Qrings) $[12,13]$ by droplet epitaxy. The technique can also produce a relatively high size uniformity of the lattice-matched nanostructures, which is advantageous to the QD laser, 
for example, because the distribution of the emission wavelength is narrow, and hence, a large optical gain is attained at the center wavelength.

Thanks to extensive efforts since the invention of this method in 1990, the quality of the nanostructures made by droplet epitaxy has been improved dramatically $[9,11,14]$. They are now highly luminescent due to small numbers of defects and impurities, and the first lasing of the lattice-matched QDs was attained recently [14-16].

This article is organized as follows. In Sec. 2, self-assembly by droplet epitaxy is described. Lasing characteristics are shown in Sec. 3. Single-photon Fourier spectroscopy for high-resolution photoluminescence (PL) measurements is described in detail in Sec. 4. Section 5 deals with the exciton Rabi oscillation of GaAs QDs. In Sec. 6, theoretical calculation and micro-PL studies of electronic states of double-Qrings are presented. The acceleration of PL by the Purcell effect due to photonic crystal micro cavities and its suppression by photonic bandgaps are described in Sec. 7. A brief summary is given in Sec. 8 .

\section{SELF-ASSEMBLY BY DROPLET EPITAXY}

Droplet epitaxy consists of two main steps as shown in Fig. 1. The first step is the fabrication of ultra-fine liquid droplets of a group-III metal with a low melting point such as $\mathrm{Ga}$ and In on a certain substrate by supplying its molecular beam in ultra-high vacuum. The second step is the formation of nanocrystals of the compound semiconductor by irradiation of the molecular beam of a group- $\mathrm{V}$ metal such as As [1].

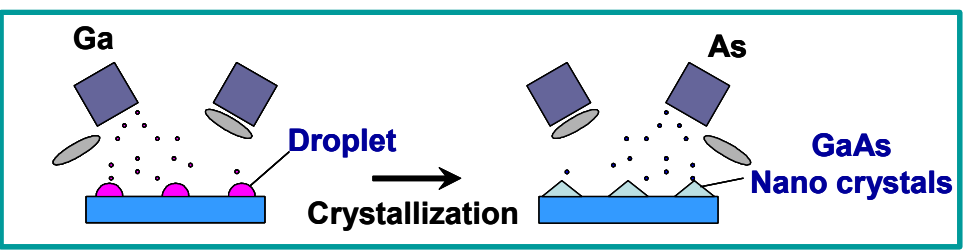

Fig. 1. Schematic illustration of droplet epitaxy.

In the initial stage of the development of droplet epitaxy, II-VI compound semiconductors such as CdTe and ZeSe were used as substrates $[1,2]$. S- or Se-terminated III-V compound semiconductors could be used as well [3]. These substrates were chosen because it is possible to avoid the growth of two-dimensional layers of the target III-V compounds and they can be made to grow three-dimensionally into epitaxial nanocrystals by suppressing the migration of the group-III metal atoms. However, it was found that the PL from these nanocrystals was weak presumably because some non-radiative recombination sites were formed between the nanocrystals and the substrate during the fabrication process, which was most probably caused by the contamination of III-V nanostructures by II-VI materials.

To solve this problem, we developed droplet epitaxy for the GaAs/AlGaAs system without using foreign materials. When we supplied Ga flux to the surface of AlGaAs epitaxial layers in the As atmosphere, of course, a two-dimensional GaAs layer was grown. But it was found later that the three-dimensional epitaxial growth of GaAs nanocrystals can be achieved in Fig. 1 by (a) supplying the Ga flux under ultra-low As pressure and (b) rapid irradiation of an intense As molecular beam [8]. For this purpose, a valved cracker cell was used as an As molecular source.

The typical self-assembly processes and the characteristics of the fabricated specimens are as follows. First, a $0.1 \mu \mathrm{m}$-thick AlGaAs barrier layer is fabricated on a GaAs (100) substrate at $580{ }^{\circ} \mathrm{C}$ by the usual MBE (molecular beam epitaxy) process. Then the substrate temperature is decreased to $200 \sim 350{ }^{\circ} \mathrm{C}$ and the pressure of the residual As atmosphere is kept below $1.3 \times 10^{-6} \mathrm{~Pa}$. Ga droplets are obtained on the substrate by supplying $\sim 4$ 
equivalent ML (mono layer) of Ga molecules at a speed of $0.5 \mathrm{ML} / \mathrm{s}$. Their average diameter is $10 \sim 50 \mathrm{~nm}$ with fluctuation of about $20 \%$. Next, As molecular flux is supplied under various conditions at $200{ }^{\circ} \mathrm{C}$ to obtain GaAs nanostructures of different shapes.

Figure 2 shows AFM (atomic force microscope) images of the substrate surface after crystallization of $\mathrm{Ga}$ droplets into GaAs by supplying the As flux with various intensities. When we supply intense As flux $\left(2.7 \times 10^{-2} \mathrm{~Pa}\right.$ beam equivalent pressure (BEP)), cone-shaped GaAs nanostructures (standard QDs) are formed due to the suppression of Ga diffusion on the substrate surface [8]. In contrast, by reducing the As flux intensity, central holes become visible. When we crystallize the droplets with an As intensity of $1.3 \times 10^{-3} \mathrm{~Pa} \mathrm{BEP}$, the central holes become very clear and well-defined single-Qrings with excellent rotational symmetry are formed $[8,11]$. Here, we would also like to mention the formation of double-QDs [10]. When we use an intermediate As intensity of $8 \times 10^{-3} \mathrm{~Pa} \mathrm{BEP}$, the shape of the nanostructures becomes highly anisotropic. They are not rings but laterally coupled double-QDs. When we further reduce the As intensity down to $1.3 \times 10^{-4} \mathrm{~Pa} \mathrm{BEP}$, another ring appears outside of the initial ring, forming double-Qrings with a well-defined central hole [12]. Hence, various GaAs epitaxial nanostructures are obtained according to each fabrication condition.

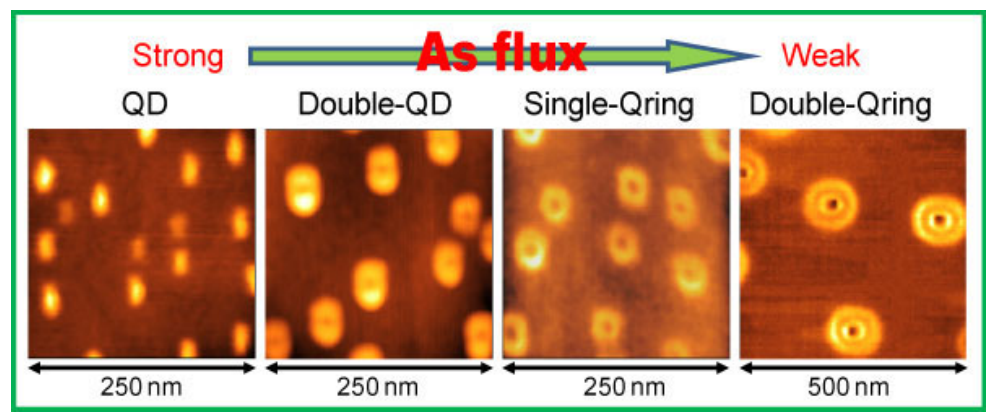

Fig. 2. Various nanostructures that can be fabricated by droplet epitaxy.

Figure 3 shows the cross-sections of some typical nanostructures measured by AFM. The lateral sizes of the single-Qrings and inner rings of double-Qrings are almost the same as the base size of the initial droplets, which may be caused by the efficient crystallization at the edges of the droplets $[11,12]$. However, we should note that the diameter of the outer ring of double-Qrings exceeds the diameter of the original Ga droplet. This indicates the migration of $\mathrm{Ga}$ atoms away from the droplet, and that of the As atoms toward the droplet.

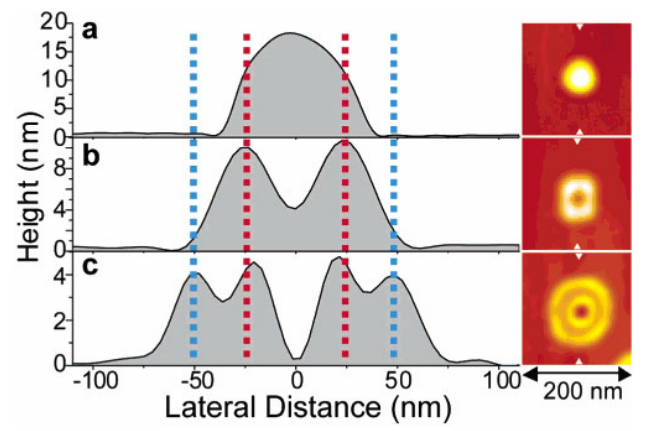

Fig. 3. AFM images and their cross-section profiles. (a) Ga droplet, (b) GaAs singleQring, (c) GaAs concentric double-Qrings. (After Ref. 12) 


\section{LASING}

As a demonstration of the good luminescent properties of our QDs, we examined the lasing characteristics [14-16]. Figure 4 shows the structure of our GaAs QD laser. Single-Qrings fabricated with As flux of $1.3 \times 10^{-3} \mathrm{~Pa}$ at $200{ }^{\circ} \mathrm{C}$ were used as active layers. Figure 5(a) shows an AFM image of the single-Qrings. Their density was $2 \times 10^{10} / \mathrm{cm}^{2}$. A cross-sectional profile of a typical QD is shown in Fig. 5(b). Their average top diameter and height are 27 and $2 \mathrm{~nm}$, respectively, with a size fluctuation of $13 \%$ in diameter and $27 \%$ in height. Figure 5(c) shows their PL spectra with a weak photo excitation. At $5 \mathrm{~K}$, an intense PL peak of carrier recombination in QDs was observed at $741 \mathrm{~nm}$, together with small signals at $682 \mathrm{~nm}$ and $688 \mathrm{~nm}$ arising from a two-dimensional GaAs layer and/or an $\mathrm{AlGaAs}$ barrier layer. The QD PL peak well fits a Gaussian function with a full width at half maximum (FWHM) of 34 $\mathrm{meV}$. The PL emission was clearly observed up to room temperature (RT) as seen in the lower panel of Fig. 5(c), which shows a doublet consisting of a major peak at $780 \mathrm{~nm}$ and a minor one at $752 \mathrm{~nm}$. The higher energy peak is related to the excited state of excitons in the QDs that were thermally activated.

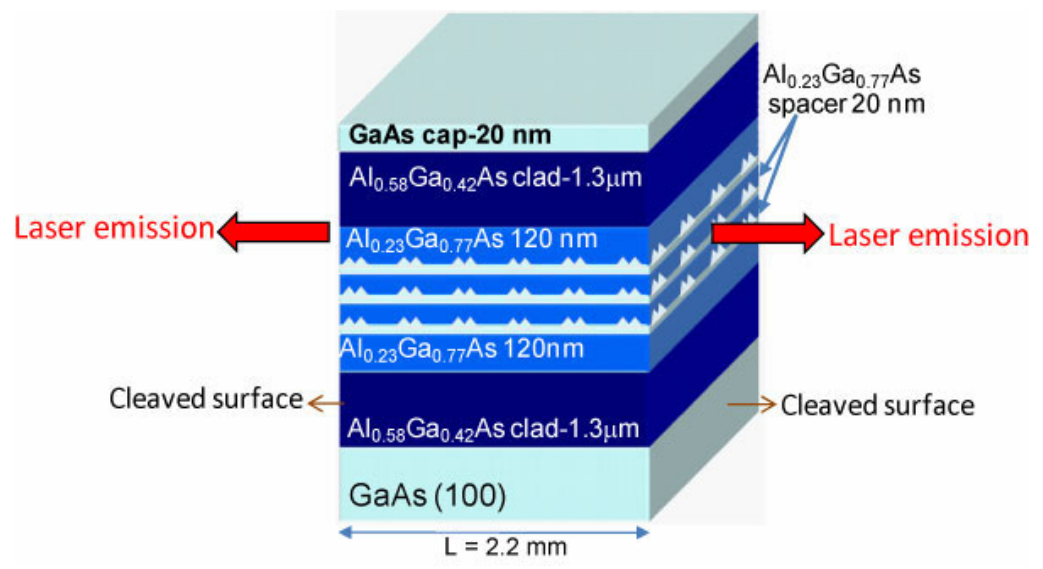

Fig. 4. Structure of the GaAs QD laser.
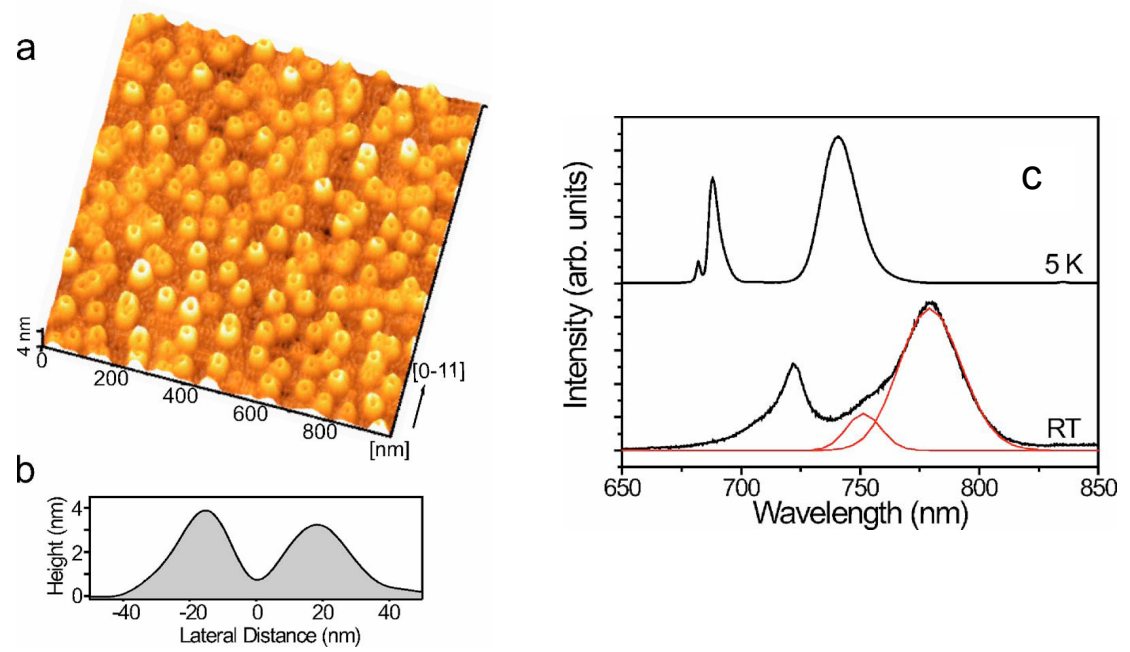

Fig. 5. (a) AFM image of GaAs single-Qrings. (b) Cross-sectional profile of a single-Qring. (c) PL spectra of an ensemble of single-Qrings measured at $5 \mathrm{~K}$ and room temperature (RT). (After Ref. 15) 
For the laser emission experiments, we used the cleaved surface as a natural laser cavity. For excitation, we used a frequency-doubled $Q$-switched neodymium yttrium aluminum garnet (Nd-YAG) laser producing pulses of $532 \mathrm{~nm}$ in wavelength, $5 \mathrm{~ns}$ in duration, and 4 $\mathrm{kHz}$ in repetition. The laser beam was focused into a single stripe on the top surface of the sample using a pair of cylindrical lenses. The width of the stripe excitation was $20 \mu \mathrm{m}$. The emission normal to the cleaved surface was detected by a spectrometer with a spectral resolution of $0.07 \mathrm{~nm}$.

Figure 6(a) shows emission spectra taken for various excitation powers at $77 \mathrm{~K}$. At low excitation below $8 \mathrm{~mW}$, the spectra show a smooth and broad curve originating from spontaneous emission of the QD ensemble. When the excitation power was increased to 12 $\mathrm{mW}$, the spectra changed into an ensemble of sharp lines originating from multimodal stimulated emission. Figure 6(b) is the excitation power dependence of the emission intensity. We can observe a clear threshold that indicates the onset of lasing.
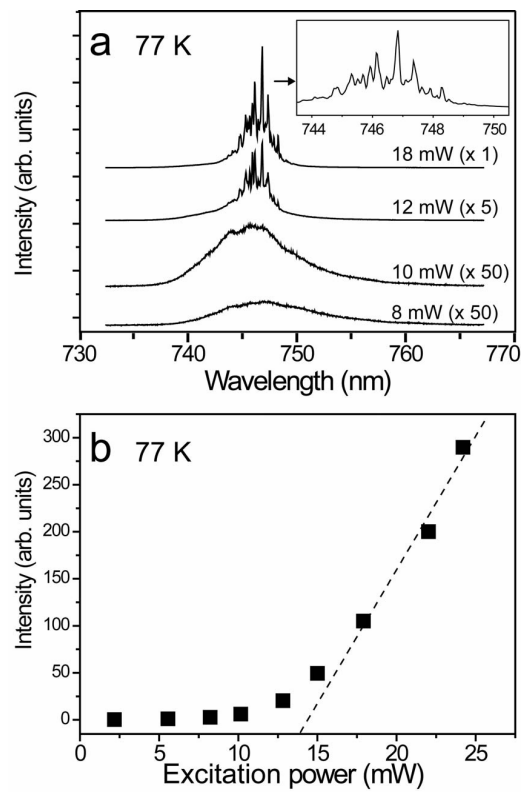

Fig. 6. (a) Emission spectra taken from the cleaved side with various excitation powers at $77 \mathrm{~K}$. (b) Emission intensities at $77 \mathrm{~K}$ as a function of the excitation power. (After Ref. 15)

We examined the temperature dependence of the lasing properties to check the quality of our device. Figure 7(a) shows the laser emission spectra at various temperatures. Since the spectral resolution of this measurement was $5 \mathrm{~nm}$, the multimodal structure was smoothed out in Fig. 7(a). Figure 7(b) is the temperature dependence of the center energy of laser emission, which shows a monotonic decrease with increasing temperature. It follows the GaAs band gap energy as shown by the dotted line in Fig. 7(b). The temperature dependence of the threshold power is shown in Fig. 7(c). Although the threshold power increased with temperature, laser action was confirmed up to RT, which is evidence for the good quality of our device. 

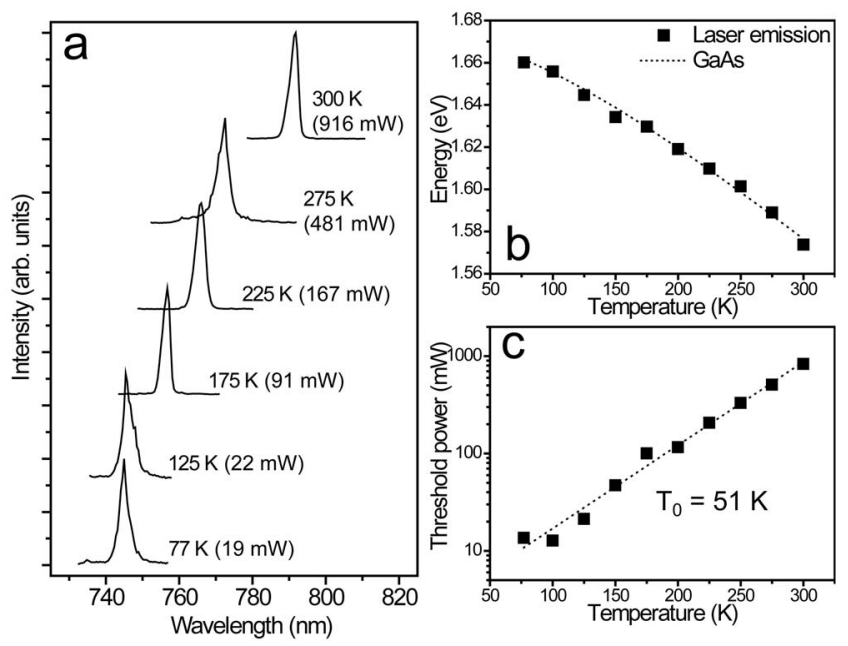

Fig. 7. (a) Lasing spectra at various temperatures. (b) Center energy and (c) threshold power of the lasing as a function of temperature. (After Ref. 15)

\section{SINGLE-PHOTON FOURIER SPECTROSCOPY OF QDS}

For accurate measurement of the emission linewidth of each QD, we used single-photon Fourier spectroscopy with a home-made stabilized autocorrelation spectrometer [17-19]. Figure 8 shows the experimental setup. We used a continuous wave (CW) He-Ne laser operating at $543 \mathrm{~nm}$ as an excitation light source. The laser beam was focused onto the specimen by a micro objective lens and the emission from the specimen was collected by the same lens. The diameter of the detection spot on the specimen was about $1.0 \mu \mathrm{m}$. The collected emission was guided to a Michelson interferometer. One of the two mirrors in the interferometer was attached to a piezoelectric transducer (PZT) mounted on a translation stage. The PZT and translation stage were used for fine and coarse tuning of the delay time, respectively. The interferometer was stabilized by a phase-locking loop (PLL) with the reflected laser beams from the two mirrors as the reference signal. This made it possible to accumulate weak single photon emissions from single QDs for a long integration time. Reflected emissions from both mirrors were carefully overlapped, dispersed by a singlegrating monochromator, and detected by a cooled charge coupled device. The monochromator was used to select a single emission line. The spectral resolution of the setup was determined by the maximum optical path difference $(30 \mathrm{~mm})$ of the Michelson interferometer, and it was $13 \mu \mathrm{eV}$. We could also measure the conventional micro-PL spectra by blocking one arm of the Michelson interferometer; the resolution for this case was $0.5 \mathrm{meV}$. Temporally resolved emission was observed by a similar micro-PL setup with the second harmonic $(400 \mathrm{~nm})$ of a mode-locked Ti:sapphire laser for excitation and a streak camera for detection.

Figure 9 shows an example of the conventional micro-PL spectrum. Sharp lines in the energy range from 1.7 to $1.85 \mathrm{eV}$ are attributed to recombination of excitons of the QDs, whereas two broad peaks around $1.95 \mathrm{eV}$ are emission from the barrier (AlGaAs) layer. The wavelength of the QD exciton emission peaks has a distribution caused by the size-dependent quantum confinement. The spectral width of each QD emission was limited by the resolution of the monochromator.

To accurately evaluate the linewidth of a single QD emission, we selected one PL line by the monochromator and observed its dependence on the optical path difference. Some examples of the output of the interferometer are shown in Fig. 10. The excitation intensity 


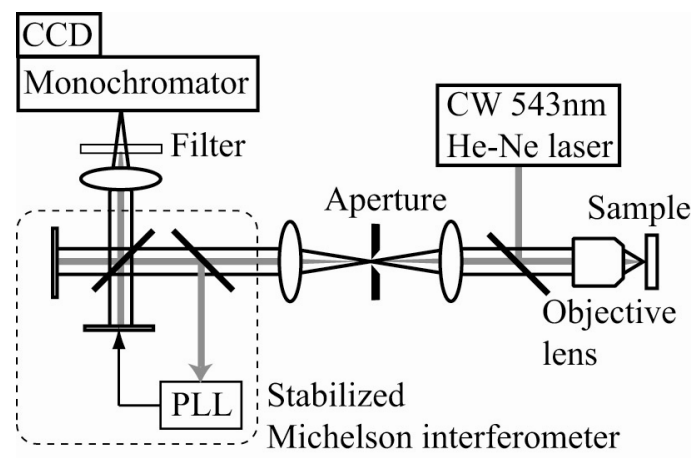

Fig. 8. Stabilized single-photon Fourier spectrometer.

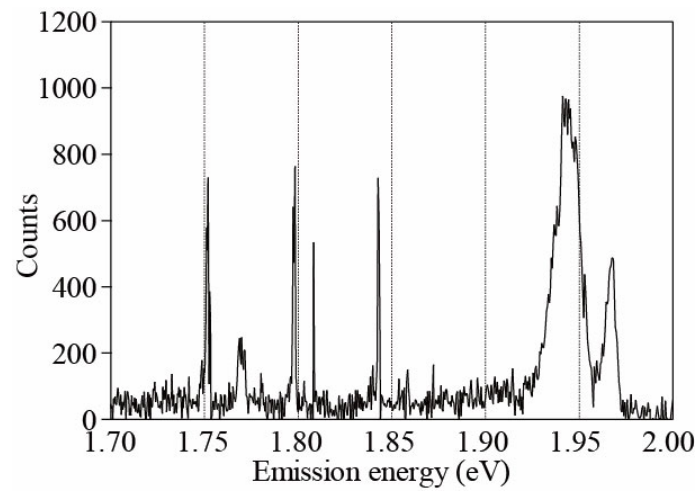

Fig. 9. Typical micro-PL spectrum of exciton emissions of GaAs QDs at $4 \mathrm{~K}$.

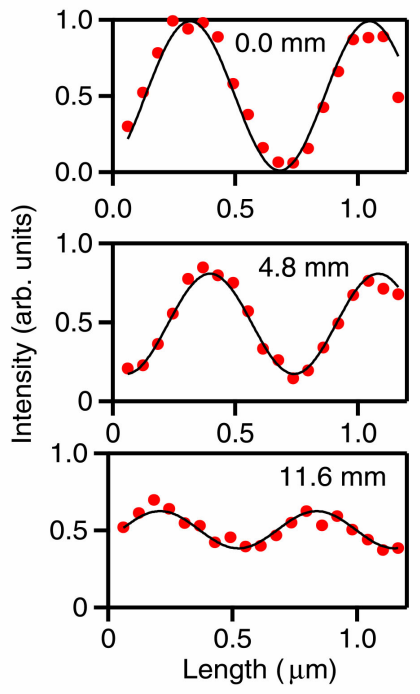

Fig. 10. Interferograms at various optical path differences. (After Ref. 17) 
was $3.2 \mathrm{~W} / \mathrm{cm}^{2}$. In this case, the average number of excitons in a QD was 0.09 . In the top panel of Fig. 10, the optical path difference varied around $0 \mathrm{~mm}$. The observed data show a continuous change in intensity between the constructive and destructive interference. The visibility of the interference is $\sim 0.97$. With increasing optical path difference, we find a decrease in the visibility $(0.66$ at $4.8 \mathrm{~mm}$ and 0.34 at $11.6 \mathrm{~mm})$, while the sinusoidal modulation with the same interval, which is determined by the emission wavelength, is still present as shown in the middle and bottom panels of Fig. 10. These results imply a finite coherence length of the electric field emitted by the single QD reflecting the phase disturbance of the excitonic dipole.

The diamonds in Fig. 11 show the delay time (= optical path difference / velocity of light) dependence of the visibility of the interferogram. The upper solid line is a fitting curve by a single exponential decay. Experimental data are well reproduced by this function, which means that the emission line shape is Lorentzian. In this example, the decoherence time of the exciton was estimated to be $35 \mathrm{ps}$, which is equivalent to a homogeneous linewidth of $38 \mu \mathrm{eV}$ (FWHM).

When the excitation density was increased to $32 \mathrm{~W} / \mathrm{cm}^{2}$, for which 0.9 excitons are excited in a dot on average, the correlation function changed to an oscillatory shape as shown by circles in Fig. 11. The solid line is a fitting curve by a model consisting of two dipoles with different frequencies. By the curve fitting, we found that the oscillation period was 4.2 ps and that the decoherence times for the slow and fast components were $32 \pm 5$ ps and $5.4 \pm 1 \mathrm{ps,}$ respectively. Note that the decoherence time of the slow component agrees well with that of the weak excitation case. For this reason, we can conclude that the slow component comes from an exciton recombination, whereas the fast component comes from a biexciton recombination, i.e., the electron-hole recombination of the two-exciton state leaving an exciton. The oscillatory profile shown in the interferogram reflects an energy difference between the excitonic and biexcitonic transitions. The inset depicts the Fourier transform of the autocorrelation function, which is a reconstructed spectrum of the single QD emission. It shows a biexcitonic satellite peak at $0.95 \mathrm{meV}$ from the exciton line at the origin. This energy difference is the biexciton binding energy.

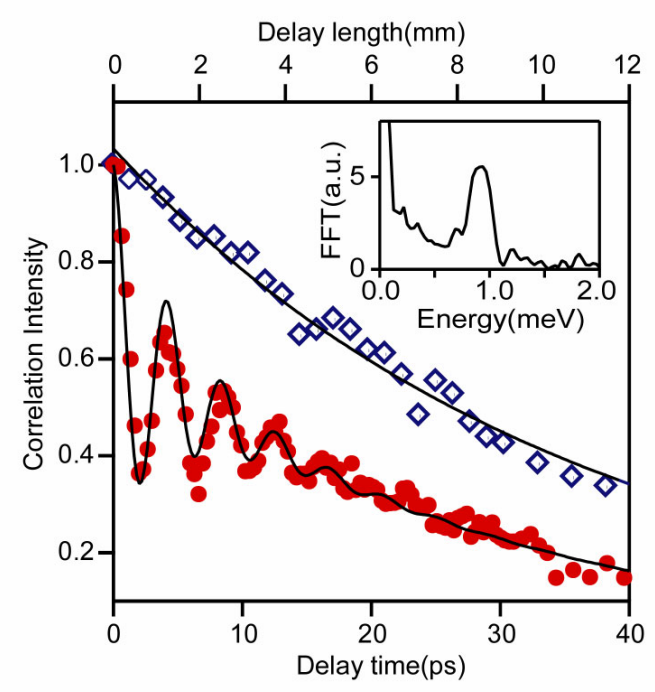

Fig. 11. Autocorrelation intensity (visibility) of the interferograms. Diamonds: weak excitation; circles: intense excitation. The inset is the Fourier transform of the visibility for the intense excitation case. (After Ref. 17) 
We measured the autocorrelation for about 40 QDs. The histogram in Fig. 12 shows the distribution of the linewidth of their exciton emission. The linewidth ranges from 40 to 400 $\mu \mathrm{eV}$ with a maximum at $70 \mu \mathrm{eV}$. The inset of Fig. 12 shows the emission energy dependence of the linewidth. We do not see a correlation between the two quantities, implying that the linewidth distribution is not caused by the size distribution of the QDs. The smallest linewidth of $40 \mu \mathrm{eV}$ does not agree with the natural linewidth of $2.9 \mu \mathrm{eV}$ obtained by the time-resolved micro-PL experiment. These results imply the presence of pure dephasing and/or spectral diffusion even at $4 \mathrm{~K}$. Especially the extremely large distribution of the linewidth is clear evidence for inhomogeneity of the local environment of QDs. In this experiment, we excited the AlGaAs barrier layer by the $543 \mathrm{~nm}$ line of a He-Ne laser to generate positive (hole) and negative (electron) photocarriers. These carriers were captured by the QD, formed an exciton, and recombined to emit a photon. So, there must be many photocarriers in the barrier layer. These charged carriers produce fluctuating electric field at the position of the QD, which results in the fluctuating Stark shift of the exciton emission. This phenomenon is a kind of spectral diffusion and is observed as a temporal shift and/or broadening of the emission line according to the observation conditions. Because the typical accumulation time of our PL experiment was five seconds, the spectral diffusion taking place in this period is included in our data and observed as a linewidth. These observations coincide with those of previous studies on InGaAs [20], CdSe [21-23], InAlAs [24], and InP QDs [25].

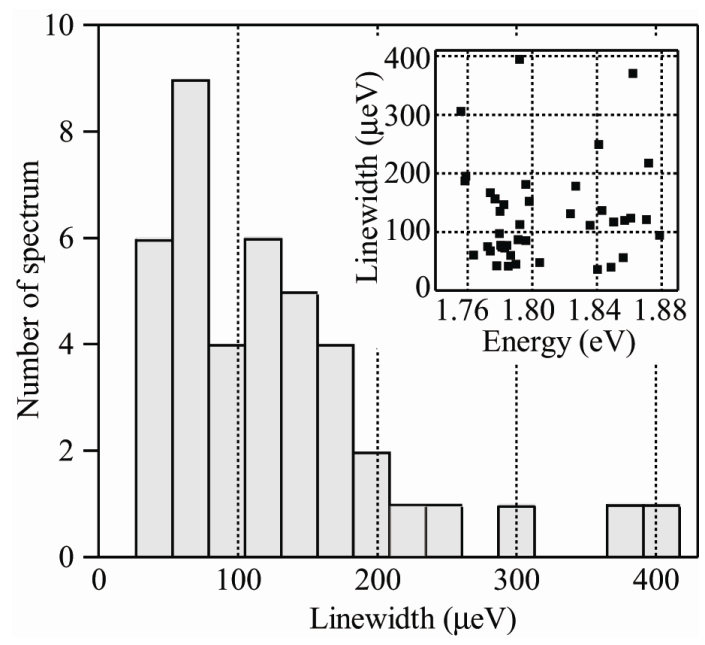

Fig. 12. Histogram of the linewidth for 40 emissions at $4 \mathrm{~K}$. Inset: Emission energy dependence of the linewidth.

\section{EXCITONIC RABI OSCILLATION OF QDS}

Because of the three-dimensional quantum confinement of wave functions, QDs have discrete electronic energy levels. So, the lowest exciton state and the ground state of the QD can often be dealt with as a two-level system in various kinds of optical processes. Especially the twolevel system is a promising candidate for a qubit (quantum bit) in quantum computation [2629], since the dephasing (decoherence) time of the QD exciton is generally long due to the decreased number of electron-phonon scattering channels [31-35], and hence, the coherence of the qubit is maintained for a sufficiently long time to repeat quantum operations.

There are two kinds of basic quantum operations, that is, one-qubit rotation and two-qubit controlled NOT operation. The latter needs optical control of two interacting QDs and is still actively being investigated. As for the former, we need to realize coherent optical transitions 
from the ground state to the exciton state and vice versa. This process is called the excitonic Rabi oscillation and has already been attained by several methods. In these experiments, the presence of the third level was necessary to monitor the electron population in the exciton state. Recently we observed the Rabi oscillation by the resonant excitation of the exciton state by femtosecond laser pulses and a successive time resolved luminescence measurement [29, 30]. This method is simpler than the previous ones because we do not need the third level and we can fully use the long decoherence time of the QD excitons. In the rest of this section, we briefly describe this experiment.

Figure 14 is the time-resolved PL emission of a single GaAs QD measured by a streak camera after excitation by a femtosecond pulsed Ti:sapphire laser. An intense signal at $0 \mathrm{ps}$ is the reflection of the excitation laser pulse. Following this instantaneous component, a decaying signal is observed, which is spontaneous emission of the single QD exciton state. The red line is a curve fitting by an exponential function and the decay time (exciton lifetime) is estimated to be $220 \pm 10 \mathrm{ps}$. The inset of Fig. 14 shows the PL spectra at 0,100 , and $300 \mathrm{ps,}$ by which we can confirm that there is no spectral change and the time-resolved signal originates from the QD exciton emission.

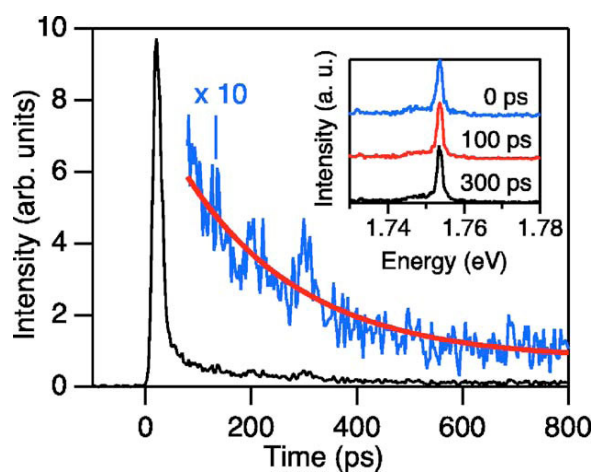

Fig. 14. Time-resolved PL emission of a single QD. The inset shows the PL spectra at 0,100 , and 300 ps. (After Ref. 29)

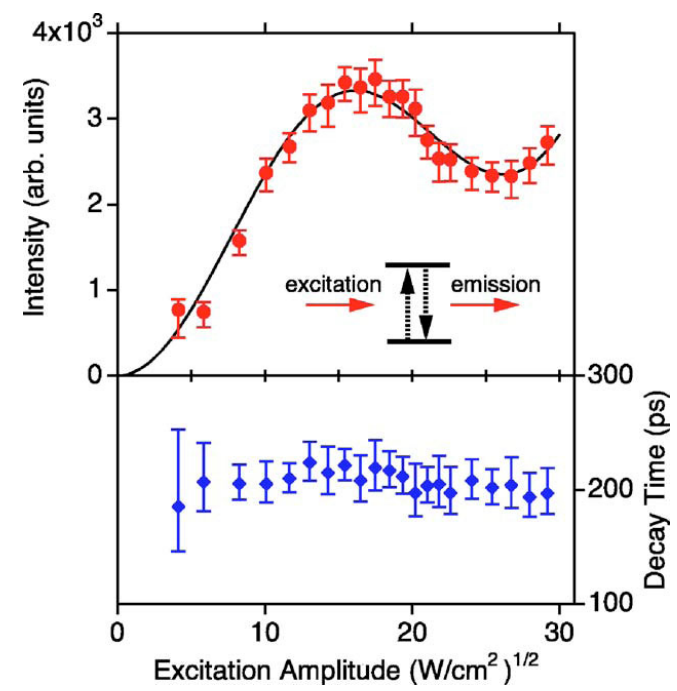

Fig. 15. Top: Excitonic Rabi oscillation measured by the time-resolved emission intensity as a function of excitation intensity. Bottom: Fluorescence lifetime for various excitation intensities. (After Ref. 29) 
When the excitation intensity was increased, the emission intensity first increased and took a maximum. Then the emission intensity decreased with increasing excitation intensity. After it took a minimum, the emission intensity increased again with increasing excitation intensity. The upper panel of Fig. 15 shows the dependence of the intensity of the single QD emission on the excitation intensity. An oscillatory behavior is clearly found. On the other hand, there is no significant change in the fluorescence lifetime as shown in the lower panel. So the oscillatory behavior is not caused by the increase in non-radiative processes which might be brought about by the intense excitation, but rather is caused by the Rabi oscillation, i.e., oscillation of the electronic population between the excited and ground states due to coherent interaction with the radiation field.

\section{ELECTRONIC STATES OF DOUBLE-QRINGS}

In this section, we show the theoretical calculation and the micro-PL measurement of exciton emission from GaAs double-Qrings [12, 13]. The results are summarized in Fig. 16. The specimen was fabricated by the method described in Sec. 2 and capped by an $\mathrm{Al}_{0.3} \mathrm{Ga}_{0.7} \mathrm{As}$ barrier layer to confine the excitons in the double-Qrings. A heat treatment called rapid thermal annealing was applied to the specimen at $750{ }^{\circ} \mathrm{C}$ for 4 minutes to reduce the number of defects and increase the optical quality.

The colored bars in the top-left part of Fig. 16 show the optical transition energies calculated with the effective mass approximation. We assumed a perfect circular symmetry for the double-ring structure around an axis perpendicular to the substrate surface, hence the energy levels of the electron and hole are characterized by the principal quantum number $N$ and the angular momentum $J$. In general, the electron and hole captured by the double-Qrings may have different $N$ and $J$. However, the theoretical calculation shows that the dominant contribution to the optical transition oscillator strength comes from the same $N$ and $J$ states for the electron and hole. So, in Fig. 16, only such transition energies are shown. Here the Coulomb attraction energy between the electron and hole is neglected, since it is smaller than the motional and confinement energies. The shape of the confinement potential, which is the same as the shape of the GaAs double rings, was given by the AFM measurement and is shown in the right-bottom part of Fig. 16. The upper three illustrations give the electron probability densities of $J=0$ states with three different $N$. The $N=0$ and 1 states are mainly located in the outer and inner rings, respectively, while the $N=2$ state has its probability density in both rings.

The micro-PL spectra measured with three different excitation intensities are presented in the left-bottom part. At low excitation intensity $\left(1 \mathrm{~W} / \mathrm{cm}^{2}\right)$, the PL consists of an intense peak at $1.68 \mathrm{eV}$ with a satellite peak located at $8 \mathrm{meV}$ on the high energy side (indicated by a blue arrow). Other minor contributions are present on the high-energy side of the main peak (indicated by red arrows), whose intensity increases with the excitation intensity. With increasing illumination, line broadening associated with the scattering of carriers, and spectral red shift that reflects multiple carrier effects are observed.

There is excellent agreement between the calculated transition energy and the PL spectra. The two distinct peaks in the PL (the main peak and the higher-energy satellite denoted by the blue arrow) are interpreted in terms of transitions of $(N, J)=(0,0)$ and $(1,0)$. The small peaks denoted by the red arrows originate from $N=0$ and $J>0$ transitions. These transitions are observed clearly under intense excitation because the $(N, J)=(0,0)$ state should be occupied to prevent fast non-radiative relaxation of $J>0$ states. 


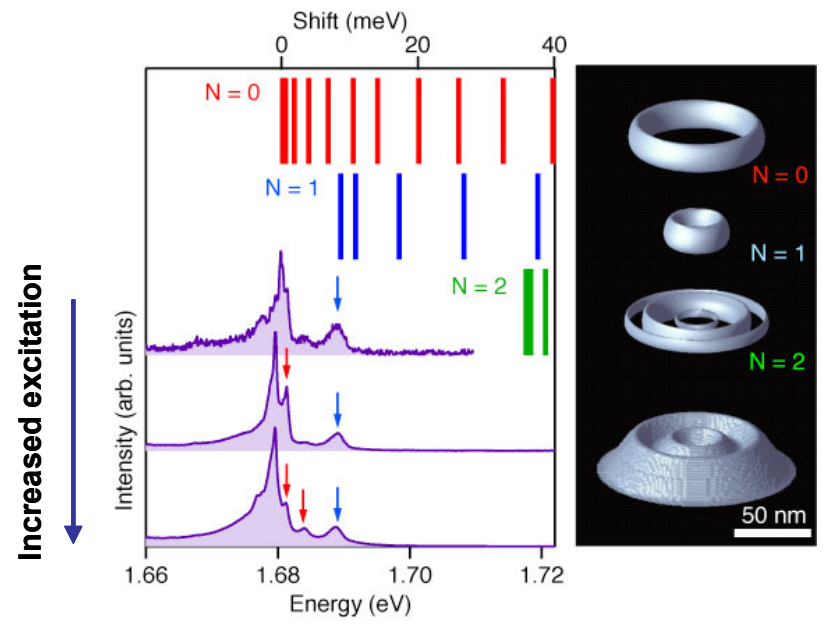

Fig. 16. Left-bottom: Three micro-PL spectra of a double-Qring measured with excitation intensity of 1,10 , and $30 \mathrm{~W} / \mathrm{cm}^{2}$. Left-top: Transition energies characterized by the principal quantum number $N$ and the angular momentum $J$. Right: Electron probability density of $J=0$ states and the potential profile used for calculation. (After Ref. 12)

\section{PURCELL EFFECT BY PC MICROCAVITIES}

In this section, we describe our recent study on the Purcell effect of GaAs QDs embedded in photonic crystal (PC) micro cavities [36]. PCs are periodic dielectric structures [37, 38]. They can create photonic bandgaps, or frequency ranges where the presence of electromagnetic eigenmodes is forbidden. So, light emission in the photonic bandgap is strongly suppressed. On the other hand, we can also create localized modes by introducing defects and disorders into the regular periodic structures of the PCs. These localized modes can be regarded as micro cavity resonators with small mode volumes. Nowadays PC micro cavities with resonance quality factors of more than one million can be fabricated. The peculiar structures that attain these large quality factors are known as air-bridged PC slabs. They are thin semiconductor plates with a thickness of the order of $1 \mu \mathrm{m}$ and usually have a regular array of air holes fabricated by electron beam (EB) or focused ion beam (FIB) lithography.

Because the electromagnetic field of the defect mode is concentrated in a small volume, it has a large amplitude in the defect region and interacts strongly with light emitters located there. One important consequence of this phenomenon is the accelerated emission of photons, which is called the Purcell effect [39]. The Purcell effect of QDs embedded in PC micro cavities has already been observed. In most of the experimental studies, InAs QDs were used for light emitters. In our case, we are interested in GaAs QDs, since their emission wavelengths are in the sensitivity range of silicon photo detectors. In future studies of coherent optical manipulation of QD wave functions to realize two-qubit quantum operations, for example, photo detectors of the highest sensitivity will be required, for which the GaAs QDs will be advantageous.

There is a scaling law between the lattice constant of the PC and the characteristic wavelengths such as those of the photonic bandgap and localized modes. Thus the lattice constant for GaAs QDs should be $40 \%$ smaller than that for InAs QDs because the emission wavelength is shorter by the same amount. In practice, PC slabs with lattice constants of around $200 \mathrm{~nm}$ are necessary to match the wavelengths of the localized defect modes to the emission wavelength of GaAs QD excitons. This was actually done using sophisticated technologies of EB lithography in our recent study [36]. 
Specimens consisting of a $0.14 \mu$ m-thick $\mathrm{Al}_{0.26} \mathrm{Ga}_{0.74} \mathrm{As}$ PC slab with a single layer of GaAs QDs in the middle plane were fabricated as follows. After a sacrificial layer of $2 \mu \mathrm{m}$ thick $\mathrm{Al}_{0.55} \mathrm{Ga}_{0.45}$ As was grown on an undoped GaAs (311)A substrate, a $0.07 \mu \mathrm{m}$-thick $\mathrm{Al}_{0.26} \mathrm{Ga}_{0.74} \mathrm{As}$ layer was made. Then GaAs QDs were formed by droplet epitaxy and covered with an $\mathrm{Al}_{0.26} \mathrm{Ga}_{0.74} \mathrm{As}$ layer. Figure 17(a) shows an AFM image of the uncovered GaAs QDs. Well-defined GaAs QDs were present with a density of $5 \times 10^{9} \mathrm{~cm}^{-2}$. Next, air-bridge PC slabs with triangular arrays of air holes were fabricated using high-resolution EB lithography, dry etching, and selective wet-etching techniques [40]. Specimens with different lattice constants were fabricated to realize PC micro cavities with various resonance frequencies. In the central part of each PC structure, an L3 cavity, which consists of three missing holes, was fabricated as shown in Fig. 17(b).
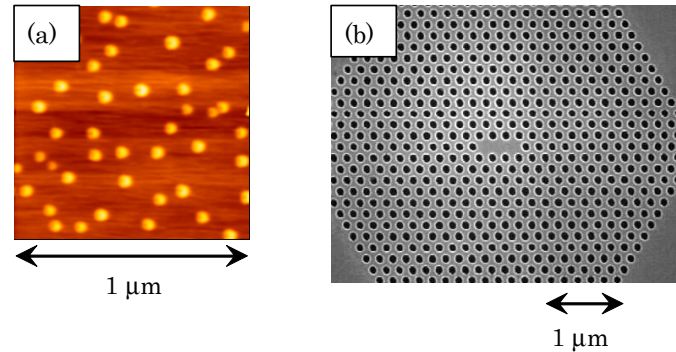

Fig. 17. (a) AFM image of GaAs QDs. (b) SEM image of the air-bridged PC slab with an L3 defect. The lattice constant is approximately $200 \mathrm{~nm}$. (After Ref. 36)
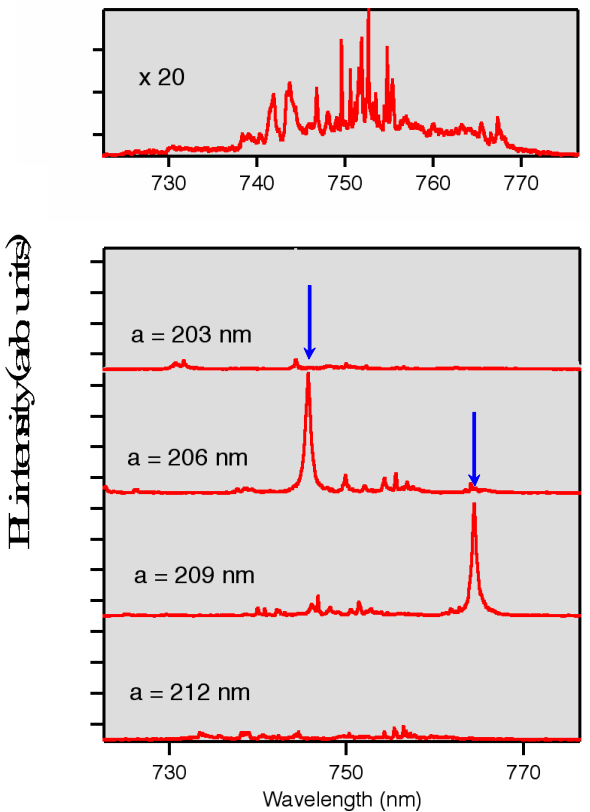

Fig. 18. Top: Micro PL spectrum of GaAs QDs without the PC structure. Bottom: Emission spectra of GaAs QDs embedded in the L3 cavities with four different lattice constants, $a$. (After Ref. 36)

The upper panel of Fig. 18 shows a micro-PL spectrum of GaAs QDs without the PC structure. It consists of sharp exciton emission peaks. The lower panel shows emission spectra 
of the QDs embedded in the PC micro cavities with four different lattice constants. As denoted by arrows, there are two big peaks for specimens with lattice constant of 206 and 209 $\mathrm{nm}$. The larger resonance wavelength for the specimen with the $209 \mathrm{~nm}$ lattice constant is consistent with the scaling law of the PC structure. The absence of the big emission peaks for specimens with lattice constant of 203 and $212 \mathrm{~nm}$ is reasonable, since the cavity resonance wavelength for these specimens is expected to be out of the emission band of the QDs.

The $\mathrm{Q}$ factor of the cavity mode, which is given by the ratio of the center frequency to the full width at half maximum, was between 500 and 2000. This value is considerably smaller than the calculated value of 5600 because of the imperfections of the PC structure that inevitably exist in actual specimens.

To confirm the acceleration of photoemission by the Purcell effect, we measured the emission lifetime with a streak camera for three cases. The first one is the genuine exciton photoemission from GaAs QDs without the PC structure, which is denoted by a black line in Fig. 19. The second case is the enhanced emission line at the resonance frequency $(746 \mathrm{~nm})$ for the specimen with lattice constant of $206 \mathrm{~nm}$ (the red curve in Fig. 19). The emission lifetime in this case was $180 \mathrm{ps}$, which is shorter than the genuine case by a factor of three. The third case is the emission line at $755 \mathrm{~nm}$, which is off resonance of the cavity mode (the blue curve in Fig. 19). We found that its lifetime was greater than 4 ns, while the emission intensity was small and the $\mathrm{S} / \mathrm{N}$ ratio of the lifetime measurement was not large. The shorter and longer lifetimes for the resonance and non-resonance cases are brought about by the acceleration of photoemission due to the Purcell effect and its deceleration by the presence of a photonic bandgap, respectively. The Purcell factor, which is given by the ratio of the genuine radiative lifetime to that of the cavity resonance case, is approximately three. This value was considerably smaller than the ideal value of 120 obtained by a theoretical calculation, which we attribute to the smaller Q due to the imperfection and the displacement of the relevant QD from the position of the maximum electric field.

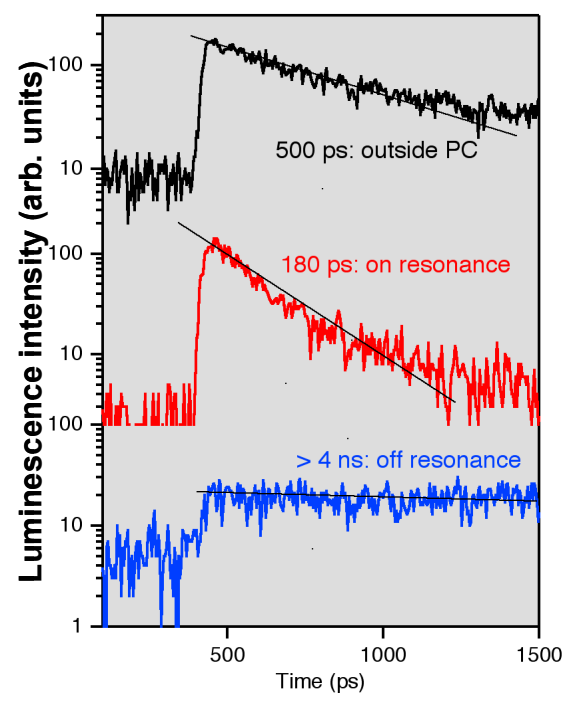

Fig. 19. Temporal variation of the emission intensity of GaAs QDs. Black: Genuine emission without the PC structure; Red: Emission at the peak frequency enhanced by the Purcell effect; Blue: Emission at the frequency in the photonic bandgap. (After Ref. 36) 


\section{SUMMARY}

In this review, we described the developments in the self-assembly of quantum nanostructures by droplet epitaxy and their electronic states and optical properties. Especially micro-PL studies on GaAs QDs and Qrings were explained in detail. In addition, we reported the first lasing of self-assembled GaAs/AlGaAs QDs.

Because the emission wavelengths of GaAs QDs are in the sensitivity range of highefficiency silicon photo detectors, they are advantageous for photon correlation measurements that may be necessary in the study of two-qubit quantum operations, for example. DoubleQrings and double-QDs can be regarded as two interacting qubits, and so they may be good candidates for the realization of two-qubit operations by optical methods.

On the other hand, when we apply a magnetic field on Qrings, field-induced phase changes in the wave functions of electrons and holes may take place. The amount of the phase change depends on the magnetic flux penetrating the ring structure. Thus, this is a kind of the Aharonov-Bohm $(\mathrm{AB})$ effect. Because excitons are neutral particles, one may think that the $\mathrm{AB}$ effect of excitons is cancelled out by the positive and negative phase changes of electrons and holes. But actually it isn't because of their different trajectories brought about by different effective mass and confinement potential.

QDs and Qrings are appropriate specimens for these investigations, which are now in progress.

\section{Acknowledgments}

We would like to express our sincere thanks to Dr. Nobuyuki Koguchi for his invention and improvement of droplet epitaxy and his guidance and collaboration on all studies described in this review article. We also thank Dr. Tetsuyuki Ochiai for the calculation of electronic levels of double-Qrings. We deeply appreciate the fabrication of air-bridge photonic crystal slabs by Dr. Kiyoshi Asakawa, Dr. Yoshimasa Sugimoto, and Dr. Naoki Ikeda. This work was supported by KAKENHI (20340080).

\section{References}

[1] N. Koguchi, S. Takahashi, and T. Chikyow, "New MBE growth method for InSb quantum well boxes," J. Cryst. Growth 111, 688-692 (1991) [doi: 10.1016/00220248(91)91064-H].

[2] T. Chikyow and N. Koguchi, "MBE growth method for pyramid-shaped GaAs micro crystals on $\mathrm{ZnSe}(001)$ surface using Ga droplets," Jpn. J. Appl. Phys. 29, L2093L2095 (1990) [doi:10.1143/JJAP.29.L2093].

[3] N. Koguchi and K. Ishige, "Growth of GaAs epitaxial microcrystals on an Sterminated GaAs substrate by successive irradiation of Ga and As molecular beams," Jpn. J. Appl. Phys. 32, 2052-2058 (1993) [doi:10.1143/JJAP.32.2052].

[4] J. Ahopelto, A. Yamaguchi, K. Nishi, A. Usui, and H. Sakaki, "Nanoscale InP islands for quantum box structures by hydride vapor phase epitaxy," Jpn. J. Appl. Phys. 32, L32-L35 (1993) [doi:10.1143/JJAP.32.L32].

[5] D. Leonard, M. Krishnamulty, C. M. Reaves, S. P. Denbaars, and P. M. Petroff, "Direct formation of quantum-sized dots from uniform coherent islands of InGaAs on GaAs surfaces," Appl. Phys. Lett. 63, 3203-3205 (1993) [doi:10.1063/1.110199].

[6] T. Mano, K. Watanabe, S. Tsukamoto, H. Fujioka, M. Oshima, and N. Koguchi, "New self-organized growth method for InGaAs quantum dots on $\mathrm{GaAs}(001)$ using droplet epitaxy," Jpn. J. Appl. Phys. 38, L1009-L1011 (1999) [doi:10.1143/JJAP.38.L1009]. 
[7] T. Mano, K. Watanabe, S. Tsukamoto, N. Koguchi, H. Fujioka, M. Oshima, C. Lee, J. Leem, H. Lee, and S. Noh, "Nanoscale InGaAs concave disks fabricated by heterogeneous droplet epitaxy," Appl. Phys. Lett. 76, 3543-3545 (2000) [doi:10.1063/1.126701].

[8] K. Watanabe, N. Koguchi, and Y. Gotoh, "Fabrication of GaAs quantum dots by modified droplet epitaxy," Jpn. J. Appl. Phys. 39, L79-L81 (2000) [doi:10.1143/JJAP.39.L79].

[9] K. Watanabe, S. Tsukamoto, Y. Gotoh, and N. Koguchi, "Photoluminescence studies of GaAs quantum dots grown by droplet epitaxy," J. Cryst. Growth 227-228, 10731077 (2001) [doi:10.1016/S0022-0248(01)00991-5].

[10] M. Yamagiwa, T. Mano, T. Kuroda, T. Takeno, K. Sakoda, G. Kido, N. Koguchi, and F. Minami, "Self-assembly of laterally aligned GaAs quantum dot pairs," Appl. Phys. Lett. 89, 113115 (2006) [doi:10.1063/1.2354007].

[11] T. Mano and N. Koguchi, "Nanometer-scale GaAs ring structure grown by droplet epitaxy," J. Cryst. Growth 278, 108-112 (2005) [doi:10.1016/j.jcrysgro.2004.12.119].

[12] T. Mano, T. Kuroda, S. Sanguinetti, T. Ochiai, T. Tateno, J. Kim, T. Noda, M. Kawabe, K. Sakoda, G. Kido, and N. Koguchi, "Self-assembly of concentric quantum double rings," Nano Lett. 5, 425-428 (2005) [doi:10.1021/nl048192+].

[13] T. Kuroda, T. Mano, T. Ochiai, S. Sanguinetti, K. Sakoda, G. Kido, and N. Koguchi, "Optical transitions in quantum-ring complexes," Phys. Rev. B 72, 205301 (2005) [doi:10.1103/PhysRevB.72.205301].

[14] T. Mano, T. Kuroda, K. Mitsuishi, M. Yamagiwa, X. -J. Guo, K. FUruya, K. Sakoda, and N. Koguchi, "Ring-shaped GaAs quantum dot laser grown by droplet epitaxy: Effects of post-growth annealing on structural and optical properties," J. Cryst. Growth 301-302, 740-743 (2007) [doi:10.1016/j.jcrysgro.2006.11.216].

[15] T. Mano, T. Kuroda, M. Yamagiwa, G. Kido, K. Sakoda, and N. Koguchi, "Lasing in GaAs/AlGaAs self-assembled quantum dots," Appl. Phys. Lett. 89, 183102 (2006) [doi:10.1063/1.2372448].

[16] T. Mano, T. Kuroda, K. Mitsuishi, Y. Nakayama, T. Noda, and K. Sakoda, "GaAs/AlGaAs quantum dot laser fabricated on GaAs(311)A substrate by droplet epitaxy," Appl. Phys. Lett. 93, 203110 (2008) [doi:10.1063/1.3026174].

[17] K. Kuroda, T. Kuroda, K. Sakoda, K. Watanabe, N. Koguchi, and G. Kido, "Excitonic and biexcitonic decoherence in self-assembled GaAs quantum dots as observed by phase-locked interferography," Appl. Phys. Lett. 88, 124101 (2006) [doi:10.1063/1.2187441].

[18] K. Kuroda, T. Kuroda, K. Sakoda, G. Kido, and N. Koguchi, "Interferometric measurement in GaAs single quantum dots: Temperature dependence of exciton $\begin{array}{lllll}\text { decoherence," } & J & \text { Lumin. } & \text { 122-123, } & \text { 789-791 }\end{array}$ [doi:10.1016/j.jlumin.2006.01.289].

[19] K. Kuroda, T. Kuroda, G. Kido, N. Koguchi, and Kazuaki Sakoda, unpublished.

[20] K. Leosson, J. R. Jensen, J. M. Hvam, and J. M. Langbein, "Linewidth statistics of single InGaAs quantum dot photoluminescence lines," phys. stat. sol. (b) 221, 49-53 (2000) [doi:10.1002/1521-3951(200009)221:1<49::AID-PSSB49>3.0.CO;2-F].

[21] V. Türck, S. Rodt, O. Stier, R. Heitz, R. Engelhardt, U. W. Pohl, D. Bimberg, and R. Steingrüber, "Effect of random field fluctuation on excitonic transitions of individual CdSe quantum dots," Phys. Rev. B 61, 9944-9947 (2000) [doi:10.1103/PhysRevB.61.9944].

[22] R. G. Neuhauser, K. T. Shimizu, W. K. Woo, S. A. Empedocles, and M. G. Bawendi, "Correlation between fluorescence intermittency and spectral diffusion in single semiconductor quantum dots," Phys. Rev. Lett. 85, $3301-3304$ (2000) [doi:10.1103/PhysRevLett.85.3301]. 
[23] J. Seufert, R. Weigand, G. Bacher, T. Kümmell, A. Forchel, K. Leonardi, and D. Hommel, "Spectral diffusion of the exciton transition in a single self-organized quantum dot," Appl. Phys. Lett. 76, 1872-1874 (2000) [doi:10.1063/1.126196].

[24] H. D. Robinson and B. B. Goldberg, "Light-induced spectral diffusion in single selfassembled quantum dots," Phys. Rev. B 61, R5086-R5089 (2000) [doi:10.1103/PhysRevB.61.R5086].

[25] P. G. Blome, M. Wenderoth, M. Hübner, R. G. Ulbrich, J. Porsche, and F. Scholz, "Temperature-dependent linewidth of single $\mathrm{InP} / \mathrm{Ga}_{\mathrm{x}} \mathrm{In}_{1-\mathrm{x}} \mathrm{P}$ quantum dots: Interaction with surrounding charge configurations," Phys. Rev. B 61, 8382-8387 (2000) [doi:10.1103/PhysRevB.61.8382].

[26] H. Kamada, H. Gotoh, J. Temmyo, T. Takagahara, and H. Ando, "Exciton Rabi oscillation in a single quantum dot," Phys. Rev. Lett. 87, 246401 (2001) [doi:10.1103/PhysRevLett.87.246401].

[27] T. H. Stievater, X. Li, D. G. Steel, D. Gammon, D. S. Katzer, D. Park, C. Piermarocchi, and L. J. Sham, "Rabi oscillations of excitons in single quantum dots," Phys. Rev. Lett. 87, 133603 (2001) [doi:10.1103/PhysRevLett.87.133603].

[28] A. Muller, Q. Q. Wang, P. Bianucci, C. K. Shih, and Q. K. Xue, "Determination of anisotropic dipole moments in self-assembled quantum dots using Rabi oscillations," Appl. Phys. Lett. 84, 981-983 (2004) [doi:10.1063/1.1646462].

[29] K. Kuroda, T. Kuroda, K. Watanabe, T. Mano, K. Sakoda, G. Kido, and N. Koguchi, "Final-state read-out of exciton qubits by observing resonantly excited photoluminescence in quantum dots," Appl. Phys. Lett. 90, 051909 (2007) [doi:10.1063/1.2435600].

[30] K. Kuroda, T. Kuroda, K. Sakoda, G. Kido, and N. Koguchi, "Exciton Rabi oscillation observed in spontaneous emissions after resonant excitation," Physica E, 40, 310-312 (2007) [doi:10.1016/j.physe.2007.06.015].

[31] D. Gammon, E. S. Snow, B. V. Shanabrook, D. S. Katzer, and D. Park, "Homogeneous linewidths in the optical spectrum of a single gallium arsenide quantum dot," Science 273, 87-90 (1996) [doi:10.1126/science.273.5271.87].

[32] K. Inoue, R. Kuribayashi, K. Sakoda, V. A. Tsekhomski, and A. V. Baranov, "Anomalous phase relaxation characteristics in $\mathrm{CuCl}$ quantum dots analogous to that in dye-molecules in polymer," phys. stat. sol. (a) 164, 437-440 (1997) [doi:10.1002/1521-396X(199711)164:1<437::AID-PSSA437>3.0.CO;2-L].

[33] R. Kuribayashi, K. Inoue, K. Sakoda, V. A. Tsekhomskii, and A. V. Baranov, "Long phase-relaxation time in $\mathrm{CuCl}$ quantum dots: Four-wave-mixing signals analogous to dye-molecules in polymers," Phys. Rev. B 57, R15084-R15087 (1998) [doi:10.1103/PhysRevB.57.R15084]

[34] R. Kuribayashi, K. Inoue, K. Sakoda, V. A. Tsekhomsii, and A. V. Barnov, "Heterodyne-detected accumulated photon-echo spectroscopy of $\mathrm{CuCl}$ quantum dots," Jpn. J. Appl. Phys. 38, 577-580 (1999) [doi:10.1143/JJAP.38.577].

[35] P. Borri, W. Langbein, S. Schneider, U. Woggon, R. L. Sellin, D. Ouyang, and D. Bimberg, "Ultralong dephasing time in InGaAs quantum dots," Phys. Rev. Lett. 87, 157401 (2001) [doi:10.1103/PhysRevLett.87.157401].

[36] D. Birkedal, K. Leosson, and J. M. Hvam, "Long lived coherence in self-assembled quantum dots," Phys. Rev. Lett. 87, $227401 \quad$ (2001) [doi:10.1103/PhysRevLett.87.227401].

[37] T. Kuroda, N. Ikeda, T. Mano, Y. Sugimoto, T. Ochiai, K. Kuroda, S. Ohkouchi, N. Koguchi, K. Sakoda, and K. Asakawa, "Acceleration and suppression of photoemission of GaAs quantum dots embedded in photonic crystal microcavities," Appl. Phys. Lett. 93, 111103 (2008) [doi:10.1063/1.2978401].

[38] J. D. Joannopoulos, R. D. Meade, and J. N. Winn, Photonic Crystals, Princeton University Press, Princeton, NJ (1995). 
[39] K. Sakoda, Optical Properties of Photonic Crystals, Springer, Berlin (2005).

[40] E. M. Purcell, "Spontaneous emission probabilities at radio frequencies," Phys. Rev. 69, 681 (1946) [doi:10.1103/PhysRev.69.37].

[41] Y. Sugimoto, N. Ikeda, N. Carlsson, K. Asakawa, N. Kawai, and K. Inoue, "Fabrication and characterization of different types of two-dimensional AlGaAs photonic crystal slabs," J. Appl. Phys. 91, 922 (2002) [doi:10.1063/1.1429795].

Takaaki Mano is a senior researcher at the Quantum Dot Research Center, National Institute for Materials Science, Japan. He received his master's degree (1999) and doctor of philosophy degree (2001) in applied chemistry from the University of Tokyo, Japan. From 1998 to 2001 he also worked at the National Institute for Materials Science in Tsukuba, Japan. For his works on the novel self-assembling growth method, he received the JSAP Incentive Award for Excellent Presentation, SSSJ Student Award, and Ishii Incentive Award. From 2001 to 2003, he was at Eindhoven University of Technology, the Netherlands, working on the ordering of self-organized nanostructures. In 2004, he returned to the National Institute for Materials Science. His current research interests are fabrication, characterization, and application of well-defined quantum confined nanostructures. For his work on self-assembly of novel quantum ring structures, he received the ICIM Takagi Award. He is a member of the Japan Society of Applied Physics.

Takashi Kuroda received his BS and $\mathrm{PhD}$ in physics from Hokkaido University in 1990 and 1994, respectively. In 1995, he joined the Tokyo Institute of Technology as a research associate, where he worked on ultrafast and nonlinear spectroscopy in semiconductors. In 2003, he joined the National Institute for Materials Science as a senior researcher, where he has been engaged in the study of semiconductor quantum dots. He is a member of the Japan Society of Applied Physics, and Physical Society of Japan.

Keiji Kuroda was a postdoctoral researcher at the Quantum Dot Research Center, National Institute for Materials Science from 2003 to 2008, and is now an assistant professor at Kitasato University, Japan. He received his BE and ME degrees in physics from Toyama University in 1995 and 1997, respectively. He received his $\mathrm{PhD}$ degree in physics from the University of Electro-Communications in 2002. His current research interests include optical studies and applications of artificial nanoscale structures. He is a member of the Japan Society of Applied Physics and the Physical Society of Japan.

Kazuaki Sakoda is the managing director of the Quantum Dot Research Center, National Institute for Materials Science, Japan. He is also a professor at the University of Tsukuba. He received his BE and ME degrees in applied physics from the University of Tokyo in 1980 and 1982, respectively. After working for TORAY Industries, Inc. as a senior researcher for 10 years, he received his $\mathrm{PhD}$ degree in applied physics from the University of Tokyo in 1992. $\mathrm{He}$ is the author of more than 100 journal papers and has written two books on photonic crystals. His current research interests include fundamental studies and application of photonic crystals and quantum confined nanostructures. He is a member of the Optical Society of America, Japan Society of Applied Physics, and Physical Society of Japan. 